\title{
Higher-Order Duality for Minimax Fractional Type Programming Involving Symmetric Matrices
}

\author{
Caiyun Jin, Cao-Zong Cheng \\ College of Applied Sciences, Beijing University of Technology, Beijing, China \\ E-mail: \{jincaiyun, czcheng\}@bjut.edu.cn \\ Received September 1, 2011; revised October 16, 2011; accepted October 23, 2011
}

\begin{abstract}
Convexity and generalized convexity play important roles in optimization theory. With the development of programming problem, there has been a growing interest in the higher-order dual problem and a lot of related generalized convexities are given. In this paper, we give the convexity of $(F, \alpha, \rho, d, b, \phi)_{\beta}$ vector-pseudoquasi-Type I and formulate a higher-order duality for minimax fractional type programming involving symmetric matrices, and give the weak, strong and strict converse duality theorems under the condition of higher-order $(F, \alpha, \rho, d, b, \phi)_{\beta}$ vector-pseudoquasi-Type I.
\end{abstract}

Keywords: Higher-Order $(F, \alpha, \rho, d, b, \phi)_{\beta}$ Vector-Pseudoquasi-Type I, Higher-Order Duality, Minimax Fractional Type Programming, Positive Semidefinite Symmetric Matrix

\section{Introduction}

In this paper, we focus on the following nondifferentiable minimax fractional programming problem:

$$
\operatorname{minsup}_{x \in R^{n}} \frac{f(x, y)+\left(x^{T} B x\right)^{\frac{1}{2}}}{h(x, y)-\left(x^{T} C x\right)^{\frac{1}{2}}}
$$

subject to

$$
g(x) \leq 0, x \in R^{n},
$$

where $Y$ is a compact subset of $R^{l}, f, h: R^{n} \times R^{l} \rightarrow R$ and $g: R^{n} \rightarrow R^{m}$ are continuously differentiable functions on $R^{n} \times R^{l}$ and $R^{n}$, respectively, and

$$
f(x, y)+\left(x^{T} B x\right)^{\frac{1}{2}} \geq 0,
$$

$h(x, y)-\left(x^{T} C x\right)^{\frac{1}{2}}>0, \forall(x, y) \in R^{n} \times R^{l}, B$ and $C$ are two positive semidefinite $n \times n$ symmetric matrices.

When $B=C=0,(P)$ is a differentiable minimax fractional programming problem.

The duality of programming problem involving symmetric matrix has been investigated widely. Schmitendorf [1] established necessary and sufficient optimality conditions for a particular case of the following problem $\left(P^{*}\right)$ under convexity conditions.

$$
\operatorname{minsup}_{y \in Y} f(x, y)+\left(x^{T} B x\right)^{\frac{1}{2}}
$$

$$
\text { subject to } \quad g(x) \leq 0 \text {. }
$$

Under the optimality conditions of [1], Tanimoto [2] defined a first-order dual problem of $\left(P^{*}\right)$, which generalized the duality theorems for convex minimax programming problems considered by Weir [3] and relaxed the convexity assumptions in the sufficient optimality of [1]. Mishra and Rueda [4] introduced generalized second-order type I functions and considered the minimax programming problem $\left(P^{*}\right)$ involving those functions and established second-order duality theorems for problem $\left(P^{*}\right)$. Husian, Anurag Jaysural and Ahmad [5] established two types of second-order dual models for problem $\left(P^{*}\right)$, which extends some previously known results on minimax programming.

With the development of programming problem, there has been a growing interest in the higher-order dual problem. Mangasarian [6] first formulated a class of secondand higher-order dual problems for a nonlinear programming problem involving twice differentiable functions. In [7], Zhang considered the following nondifferentiable mathematical programming problem:

$$
\text { Minimize } f(x)+\left(x^{T} B x\right)^{\frac{1}{2}}
$$

subject to

$$
g(x) \geq 0,
$$

under higher-order invexity assumptions. Mishra and Rueda [8] generalized the results of Zhang [7] to higher- 
order type I functions.

In [9], Ahmad, Husain and Sharma considered the nondifferentiable minimax programming problem $\left(P^{*}\right)$, and formulated a unified higher-order dual of $\left(P^{*}\right)$, and established weak, strong and strict converse duality theorems under higher-order $(F, \alpha, \rho, d)$-Type I assumptions. In [10], Jayswal and Stancu-Minasian formulated the weak, strong and strict converse duality of $\left(P^{*}\right)$ under generalized convexity of higher-order $(F, \alpha, \rho, d)$ Type I.

For problem $(P)$, H. C. Lai and K. Tanaka gave the necessary and sufficient conditions under the conditions of pseudo-convex, strictly pseudo-convex and quasi-convex [11].

In this paper, we will establish a higher-order dual of (P) and give the weak, strong and strict converse duality theorems under $(F, \alpha, \rho, d, b, \phi)_{\beta}$ vector-pseudoquasiType I assumptions. The convexity conditions in this paper generalized the convexity in [8], and hence, presents an answer of a question raised in [10].

\section{Preliminaries}

Let $R^{n}$ be the $n$-dimensional Euclidean space, $R_{+}^{n}$ be its nonegative orthant and $X$ be an open subset of $R^{n}$. Let $S$ be the set of all feasible solutions of $(P)$. Denote $M=\{1,2, \cdots, m\}$. For each $(x, y) \in S \times Y$, we define

$$
\begin{gathered}
J(x)=\left\{j \in M: g_{j}(x)=0\right\} \\
Y(x)=\left\{y \in Y: \frac{f(x, y)+\left(x^{T} B x\right)^{\frac{1}{2}}}{h(x, y)-\left(x^{T} C x\right)^{\frac{1}{2}}}\right. \\
\left.=\sup _{z \in Y} \frac{f(x, z)+\left(x^{T} B x\right)^{\frac{1}{2}}}{h(x, z)-\left(x^{T} C x\right)^{\frac{1}{2}}}\right\} \\
K(x)=\left\{(s, t, \tilde{y}) \in N \times R_{+}^{s} \times R^{l s}: 1 \leq s \leq n+1,\right. \\
t=\left(t_{1}, t_{2}, \cdots, t_{s}\right) \in R_{+}^{s} \text { with } \\
\left.\sum_{i=1}^{s} t_{i}=1, \tilde{y}=\left(\bar{y}_{1}, \bar{y}_{2}, \cdots, \bar{y}_{s}\right) \text { and } \bar{y}_{i} \in Y(x), i=1,2, \cdots, s\right\}
\end{gathered}
$$

Definition 1: A function $F: X \times X \times R^{n} \rightarrow R$ is said to be sublinear in its third argument, if $\forall x, \bar{X} \in X$,

1) (subadditivity) $\forall a_{1}, a_{2} \in R^{n}$,

$$
F\left(x, \bar{x} ; a_{1}+a_{2}\right) \leq F\left(x, \bar{x} ; a_{1}\right)+F\left(x, \bar{x} ; a_{2}\right) ;
$$

2) (positive homogeneous) $\forall \alpha \in R_{+}, a \in R^{n}$,

$$
F(x, \bar{x} ; \alpha a)=\alpha F(x, \bar{x} ; a) .
$$

Let $f: R^{n} \times R^{l} \rightarrow R, \rho=\left(\rho_{1}, \rho_{2}\right) \in R^{2}, \beta \in R^{n}$, $\alpha_{1}, \alpha_{2}: X \times X \rightarrow R_{+} \backslash\{0\}, b, d: X \times X \rightarrow R$, $e: R^{n} \rightarrow R$ and $\phi: R \rightarrow R$. Let $w: R^{n} \times R^{l} \times R^{n} \rightarrow R$, $l: R^{n} \times R^{n} \rightarrow$ and $k: R^{n} \times R^{n} \rightarrow R^{m}$ be three differentiable functions. We assume that $F$ is a sublinear functional throughout this paper.

Definition 2: $(f, e)$ is said to be higher-order $(F, \alpha, \rho, d, b, \phi)_{\beta}$ vector-pseudoquasi-Type I at $\bar{x} \in X$ with respect to $p \in R^{n}$, if for all $x \in S$ and $\bar{y} \in Y(x)$,

$$
\begin{aligned}
& b(x, \bar{x}) \phi\left[f(x, \bar{y})+x^{T} \beta-f(\bar{x}, \bar{y})-\bar{x}^{T} \beta-w(\bar{x}, \bar{y}, p)\right. \\
& \left.+p^{T} \nabla_{p} w(\bar{x}, \bar{y}, p)\right]<0 \\
& \Rightarrow F\left(x, \bar{x}, \alpha_{1}(x, \bar{x})\left(\nabla_{p} w(\bar{x}, \bar{y}, p)+\beta\right)\right) \\
& <-\rho_{1} d^{2}(x, \bar{x}) \\
& -\left[e(\bar{x})+l(\bar{x}, p)-p^{T} \nabla_{p} l(\bar{x}, p)\right] \leq 0 \\
& \Rightarrow F\left(x, \bar{x}, \alpha_{2}(x, \bar{x})\left(\nabla_{p} l(\bar{x}, p)\right)\right) \leq-\rho_{2} d^{2}(x, \bar{x}) .
\end{aligned}
$$

Definition 3: $(f, e)$ is said to be strictly higher-order $(F, \alpha, \rho, d, b, \phi)_{\beta}$ vector-pseudoquasi-Type I at $\bar{x} \in X$ with respect to $p \in R^{n}$, if for all $\bar{x} \neq x \in S$ and $\bar{y} \in Y(x)$,

$$
\begin{aligned}
& b(x, \bar{x}) \phi\left[f(x, \bar{y})+x^{T} \beta-f(\bar{x}, \bar{y})-\bar{x}^{T} \beta-w(\bar{x}, \bar{y}, p)\right. \\
& \left.+p^{T} \nabla_{p} w(\bar{x}, \bar{y}, p)\right] \leq 0 \\
& \Rightarrow F\left(x, \bar{x}, \alpha_{1}(x, \bar{x})\left(\nabla_{p} w(\bar{x}, \bar{y}, p)+\beta\right)\right) \\
& <-\rho_{1} d^{2}(x, \bar{x}) \\
& -\left[e(\bar{x})+l(\bar{x}, p)-p^{T} \nabla_{p} l(\bar{x}, p)\right] \leq 0 \\
& \Rightarrow F\left(x, \bar{x}, \alpha_{2}(x, \bar{x})\left(\nabla_{p} l(\bar{x}, p)\right)\right) \leq-\rho_{2} d^{2}(x, \bar{x}) .
\end{aligned}
$$

Obviously, when $\phi$ is subadditive function and satisfies $a \leq 0 \Rightarrow \phi(a) \leq 0$, higher-order

$(F, \alpha, \rho, d, b, \phi)_{B u}$ vector-pseudoquasi-Type I is the convexity condition of Theorem 3.1 in [10].

In the following section, we will use Lemma 1 and Lemma 2 which were given in [11].

Lemma 1: (Necessary Condition) If $x^{*}$ is an optimal solution of problem $(P)$ satisfying $x^{* T} B x^{*}>0, x^{* T} C x^{*}>0$ and $\nabla g_{j}\left(x^{*}\right), j \in J\left(x^{*}\right)$ are linear independent, then there exist $\left(s^{*}, t^{*}, \bar{y}^{*}\right) \in K\left(x^{*}\right)$, $u^{*}, v^{*} \in R^{n}$ and $\mu^{*} \in R_{+}^{m}, \lambda^{*} \in R_{+}$such that

$$
\begin{aligned}
& \sum_{i=1}^{s^{*}} t_{i}^{*}\left\{\nabla f\left(x^{*}, \bar{y}_{i}^{*}\right)-\lambda^{*}\left(\nabla h\left(x^{*}, \bar{y}_{i}^{*}\right)\right)+B u^{*}+\lambda^{*} C v^{*}\right\} \\
& +\nabla \sum_{j=1}^{m} \mu_{j}^{*} g_{j}\left(x^{*}\right)=0 \\
& f\left(x^{*}, \bar{y}_{i}^{*}\right)+\left(x^{* T} B x^{*}\right)^{\frac{1}{2}}-\lambda^{*} h\left(x^{*}, \bar{y}_{i}^{*}\right)+\lambda^{*}\left(x^{* T} C x^{*}\right)^{\frac{1}{2}}=0 \\
& i=1,2, \cdots, s^{*} \\
& \sum_{i=1}^{m} \mu_{j}^{*} g_{j}\left(x^{*}\right)=0
\end{aligned}
$$




$$
\begin{gathered}
\sum_{j=1}^{s^{*}} t_{i}^{*}=1, t_{i}^{*} \geq 0, i=1,2, \cdots, s^{*} \\
\left\{\begin{array}{c}
u^{* T} B u^{*} \leq 1, v^{* T} C v^{*} \leq 1, \\
x^{* T} B u^{*}=\left(x^{* T} B x^{*}\right)^{\frac{1}{2}}, \\
x^{* T} C v^{*}=\left(x^{* T} B x^{*}\right)^{\frac{1}{2}} .
\end{array}\right.
\end{gathered}
$$

Lemma 2: Let $B$ be a positive semidefinite symmetric matrix of order $n$. Then for all $x, u \in R^{n}$,

$$
x^{T} B u \leq\left(x^{T} B x\right)^{\frac{1}{2}} \times\left(u^{T} B u\right)^{\frac{1}{2}} .
$$

The equality holds when $B x=\gamma B u$ for some $\gamma \geq 0$. Evidently, if $\left(u^{T} B u\right)^{\frac{1}{2}} \leq 1$, we have

$$
x^{T} B u \leq\left(x^{T} B x\right)^{\frac{1}{2}} .
$$

\section{Duality Model}

We consider the following dual model (WD) .

$$
\max _{(s, t, \tilde{y}) \in K(z)} \sup _{(z, u, v, \lambda, \mu, p) \in H(s, t, \tilde{y})} \lambda,
$$

where $H(s, t, \tilde{y})$ denote the set of all

$(z, u, v, \lambda, \mu, p) \in R^{n} \times R^{n} \times R^{n} \times R_{+} \times R_{+}^{m} \times R^{n} \quad$ satisfying

$$
\begin{gathered}
\sum_{i=1}^{s} t_{i} \nabla_{p} w\left(z, \bar{y}_{i}, p\right)+B u+\lambda C v+\sum_{j=1}^{m} \nabla_{p}\left(\mu_{j} k_{j}(z, p)\right)=0 \\
u^{T} B u \leq 1, v^{T} C v \leq 1 \\
\sum_{i=1}^{s} t_{i}\left\{f\left(z, \bar{y}_{i}\right)-\lambda h\left(z, \bar{y}_{i}\right)\right\}+z^{T} B u+\lambda z^{T} C v \\
+\sum_{i=1}^{s} t_{i}\left[w\left(z, \bar{y}_{i}, p\right)-p^{T} \nabla_{p} w\left(z, \bar{y}_{i}, p\right)\right] \geq 0 \\
\sum_{j=1}^{m}\left\{\mu_{j} g_{j}(z)+\mu_{j} k_{j}(z, p)-p^{T} \nabla_{p}\left(\mu_{j} k_{j}(z, p)\right)\right\} \geq 0
\end{gathered}
$$

If for a triplet $(s, t, \tilde{y}) \in K(z)$, the set $H(s, t, \tilde{y})=\varnothing$, then we define the supremum over $H(s, t, \tilde{y})$ to be $-\infty$.

Next, we establish the duality of type (WD).

Theorem 3.1 (Weak Duality) Let $x$ and $(z, u, v, \lambda, \mu, s, t, \tilde{y}, p)$ be feasible solutions of $(P)$ and (WD), respectively. Assume that

1) $\left[\sum_{i=1}^{s} t_{i}\left(f\left(\cdot, \bar{y}_{i}\right)-\lambda h\left(\cdot, \bar{y}_{i}\right)\right), \sum_{j=1}^{m} \mu_{j} g_{j}(\cdot)\right]$ is higher-order $(F, \alpha, \rho, d, b, \phi)_{B u+\lambda C v}$ vector-pseudoquasi Type I at $z$,

2) $\phi(a) \geq 0 \Rightarrow a \geq 0, \quad b(x, z)>0$, $\frac{\rho_{1}}{\alpha_{1}(x, z)}+\frac{\rho_{2}}{\alpha_{2}(x, z)} \geq 0$.

Then

$$
\sup _{y \in Y} \frac{f(x, y)+\left(x^{T} B x\right)^{\frac{1}{2}}}{h(x, y)-\left(x^{T} C x\right)^{\frac{1}{2}}} \geq \lambda
$$

Proof: From (3.14), we know that

$$
-\left[\sum_{j=1}^{m}\left\{\mu_{j} g_{j}(z)+\mu_{j} k_{j}(z, p)-p^{T} \nabla_{p}\left(\mu_{j} k_{j}(z, p)\right)\right\}\right] \leq 0,
$$

then follows form 1) and $\alpha_{2}(x, z)>0$, we have

$$
F\left(x, z ; \sum_{j=1}^{m} \nabla_{p}\left(\mu_{j} k_{j}(z, p)\right)\right) \leq-\frac{\rho_{2}}{\alpha_{2}(x, z)} d^{2}(x, z) .
$$

Since $F$ is sublinear in its third argument, by (3.11) we can get

$$
\begin{aligned}
& 0=F\left(x, z ; \sum_{i=1}^{s} t_{i} \nabla_{p} w\left(z, \bar{y}_{i}, p\right)+B u\right. \\
&\left.+\lambda C v+\sum_{j=1}^{m} \nabla_{p}\left(\mu_{j} k_{j}(z, p)\right)\right) \\
&=F\left(x, z ; \sum_{i=1}^{s} t_{i} \nabla_{p} w\left(z, \bar{y}_{i}, p\right)+B u+\lambda C v\right) \\
& \quad+F\left(x, z ; \sum_{j=1}^{m} \nabla_{p}\left(\mu_{j} k_{j}(z, p)\right)\right) \\
& \leq F\left(x, z ; \sum_{i=1}^{s} t_{i} \nabla_{p} w\left(z, \bar{y}_{i}, p\right)+B u+\lambda C v\right) \\
& \quad-\frac{\rho_{2}}{\alpha_{2}(x, z)} d^{2}(x, z) .
\end{aligned}
$$

Furthermore, by $\frac{\rho_{1}}{\alpha_{1}(x, z)}+\frac{\rho_{2}}{\alpha_{2}(x, z)} \geq 0$ and $\alpha_{1}(x, z)>0$, we have

$$
\begin{aligned}
& F\left(x, z ; \alpha_{1}(x, z)\left(\sum_{i=1}^{s} t_{i} \nabla_{p} w\left(z, \bar{y}_{i}, p\right)+B u+\lambda C v\right)\right) \\
& \geq-\rho_{1} d^{2}(x, z),
\end{aligned}
$$

which implies that

$$
\begin{aligned}
b(x, z) \phi & \left(\left(\sum_{i=1}^{s} t_{i}\left\{f\left(x, \bar{y}_{i}\right)-\lambda h\left(x, \bar{y}_{i}\right)\right\}+x^{T} B u+\lambda x^{T} C v\right)\right. \\
& -\left(\sum_{i=1}^{s} t_{i}\left\{f\left(z, \bar{y}_{i}\right)-\lambda h\left(z, \bar{y}_{i}\right)\right\}+z^{T} B u+\lambda\left(z^{T} C v\right)\right) \\
& \left.-\sum_{i=1}^{s} t_{i}\left[w\left(z, \bar{y}_{i}, p\right)-p^{T} \nabla_{p} w\left(z, \bar{y}_{i}, p\right)\right]\right) \\
\geq & 0 .
\end{aligned}
$$


From 2) and (3.13), we can get

$$
\begin{aligned}
& \sum_{i=1}^{s} t_{i}\left\{f\left(x, \bar{y}_{i}\right)-\lambda h\left(x, \bar{y}_{i}\right)\right\}+x^{T} B u+\lambda x^{T} C v \\
& \geq \sum_{i=1}^{s} t_{i}\left\{f\left(z, \bar{y}_{i}\right)-\lambda h\left(z, \bar{y}_{i}\right)\right\}+z^{T} B u+\lambda\left(z^{T} C v\right) \\
& \quad+\sum_{i=1}^{s} t_{i}\left[w\left(z, \bar{y}_{i}, p\right)-p^{T} \nabla_{p} w\left(z, \bar{y}_{i}, p\right)\right] \\
& \geq 0 .
\end{aligned}
$$

Therefore, following from (3.12) and Lemma 2,

$$
\begin{aligned}
& \sum_{i=1}^{s} t_{i}\left[f\left(x, \bar{y}_{i}\right)+\left(x^{T} B x\right)^{\frac{1}{2}}-\lambda\left(h\left(x, \bar{y}_{i}\right)-\left(x^{T} C x\right)^{\frac{1}{2}}\right)\right] \\
& \geq \sum_{i=1}^{s} t_{i}\left\{f\left(x, \bar{y}_{i}\right)-\lambda h\left(x, \bar{y}_{i}\right)\right\}+x^{T} B u+\lambda x^{T} C v \\
& \geq 0 .
\end{aligned}
$$

Since $t=\left(t_{1}, t_{2}, \cdots, t_{s}\right) \neq 0, t_{i} \geq 0, i=1,2, \cdots, s, \bar{y}_{i} \in Y$ and $h(x, y)-\left(x^{T} C x\right)^{\frac{1}{2}}>0, \forall(x, y) \in R^{n} \times R^{l}$, at least exists one $q \in\{1,2, \cdots, s\}$, such that

$$
\frac{f\left(x, \bar{y}_{q}\right)+\left(x^{T} B x\right)^{\frac{1}{2}}}{h\left(x, \bar{y}_{q}\right)-\left(x^{T} C x\right)^{\frac{1}{2}}} \geq \lambda,
$$

which implies that

$$
\sup _{y \in Y} \frac{f(x, y)+\left(x^{T} B x\right)^{\frac{1}{2}}}{h(x, y)-\left(x^{T} C x\right)^{\frac{1}{2}}} \geq \lambda .
$$

Theorem 3.2 (Strong duality) Let $x^{*}$ be an optimal solution of (P) satisfying $x^{* T} B x^{*}>0, x^{* T} C x^{*}>0$ and let $\nabla g_{j}\left(x^{*}\right), j \in J\left(x^{*}\right)$ be linear independent. Assume that for any $i=1,2, \cdots, s^{*}$

$$
\begin{gathered}
w\left(x^{*}, \bar{y}_{i}^{*}, 0\right)=0, \\
\nabla_{p} w\left(x^{*}, \bar{y}_{i}^{*}, 0\right)=\nabla f\left(x^{*}, \bar{y}_{i}^{*}\right)-\lambda^{*}\left(\nabla h\left(x^{*}, \bar{y}_{i}^{*}\right)\right),
\end{gathered}
$$

and for any $j \in J\left(x^{*}\right)$

$$
k_{j}\left(x^{*}, 0\right)=0, \quad \nabla_{p} k_{j}\left(x^{*}, 0\right)=\nabla g_{j}\left(x^{*}\right) .
$$

Then there exist $\left(s^{*}, t^{*}, \tilde{y}^{*}\right) \in K\left(x^{*}\right)$ and $\left(x^{*}, u^{*}, v^{*}, \lambda^{*}, \mu^{*}, p^{*}\right) \in H\left(s^{*}, t^{*}, \tilde{y}^{*}\right)$ such that $\left(x^{*}, u^{*}, v^{*}, \lambda^{*}, \mu^{*}, s^{*}, t^{*}, \tilde{y}^{*}, p^{*}=0\right)$ is a feasible solution of (WD) and the two objectives have the same values. Furthermore, if the assumptions of weak duality hold for all feasible solutions of $(P)$ and (WD), then $\left(x^{*}, u^{*}, v^{*}, \lambda^{*}, \mu^{*}, s^{*}, t^{*}, \tilde{y}^{*}, p^{*}=0\right)$ is an optimal solutions of (WD).

Proof: Since $x^{*}$ is an optimal solution of (P) satisfying $x^{* T} B x^{*}>0, x^{* T} C x^{*}>0$ and $\nabla g_{j}\left(x^{*}\right), j \in J\left(x^{*}\right)$ is linearly independent, by Lemma 1 , there exist $\left(s^{*}, t^{*}, \tilde{y}^{*}\right) \in K\left(x^{*}\right), \quad u^{*}, v^{*} \in R^{n}$ and $\mu^{*} \in R_{+}^{m}, \lambda^{*} \in R_{+}$ such that

$$
\begin{gathered}
\sum_{i=1}^{s^{*}} t_{i}^{*}\left\{\nabla f\left(x^{*}, \bar{y}_{i}^{*}\right)-\lambda^{*}\left(\nabla h\left(x^{*}, \bar{y}_{i}^{*}\right)\right)+B u^{*}+\lambda^{*} C v^{*}\right\} \\
+\nabla \sum_{j=1}^{m} \mu_{j}^{*} g_{j}\left(x^{*}\right)=0 \\
f\left(x^{*}, \bar{y}_{i}^{*}\right)+\left(x^{* T} B x^{*}\right)^{\frac{1}{2}}-\lambda^{*} h\left(x^{*}, \bar{y}_{i}^{*}\right)+\lambda^{*}\left(x^{* T} C x^{*}\right)^{\frac{1}{2}}=0 \\
i=1,2, \cdots, s^{*} \\
\sum_{i=1}^{m} \mu_{j}^{*} g_{j}\left(x^{*}\right)=0 \\
\sum_{j=1}^{s^{*}} t_{i}^{*}=1, t_{i}^{*} \geq 0, i=1,2, \cdots, s^{*} \\
\left\{\begin{array}{l}
u^{* T} B u^{*} \leq 1, v^{* T} C v^{*} \leq 1 \\
x^{* T} B u^{*}=\left(x^{* T} B x^{*}\right)^{\frac{1}{2}} \\
x^{* T} C v^{*}=\left(x^{* T} B x^{*}\right)^{\frac{1}{2}}
\end{array}\right.
\end{gathered}
$$

By (2.1) (2.2) (2.3) (2.5) and the conditions of theorem 3.2, we know that $\left(x^{*}, u^{*}, v^{*}, \lambda^{*}, \mu^{*}, p^{*}=0\right) \in H\left(s^{*}, t^{*}, \tilde{y}^{*}\right)$, that is $\left(x^{*}, u^{*}, v^{*}, \lambda^{*}, \mu^{*}, s^{*}, t^{*}, \tilde{y}^{*}, p^{*}=0\right)$ is an feasible solutions of (WD). Furthermore, (3.2) implies that $\left(x^{*}, u^{*}, v^{*}, \lambda^{*}, \mu^{*}, s^{*}, t^{*}, \tilde{y}^{*}, p^{*}=0\right)$ is an optimal solutions of (WD).

Theorem 3.3 (Strict Converse Duality) Let $x^{*}$ be a feasible solution of $(P)$ and $\left(z^{*}, u^{*}, v^{*}, \lambda^{*}, \mu^{*}, p^{*}\right)$ be a feasible solution of (WD). Suppose that

$1)^{\prime}$

$$
\sup _{y^{*} \in Y} \frac{f\left(x^{*}, y^{*}\right)+\left(x^{* T} B x^{*}\right)^{\frac{1}{2}}}{h\left(x^{*}, y^{*}\right)-\left(x^{* T} C x^{*}\right)^{\frac{1}{2}}}=\lambda^{*}
$$

2)' $\left[\sum_{i=1}^{s^{*}} t_{i}^{*}\left(f\left(\cdot, \bar{y}_{i}^{*}\right)-\lambda^{*} h\left(\cdot, \bar{y}_{i}^{*}\right)\right), \sum_{j=1}^{m} \mu_{j}^{*} g_{j}(\cdot)\right]$ is strictly higher-order $(F, \alpha, \rho, d, b, \phi)_{\mathrm{Bu}^{*}+\lambda^{*} \mathrm{Cv}^{*}}$ vector-pseudoquasiType I at $z^{*}$ and;

3)' $\phi(a)>0 \Rightarrow a>0, \quad b\left(x^{*}, z^{*}\right)>0$,

$$
\frac{\rho_{1}}{\alpha_{1}\left(x^{*}, z^{*}\right)}+\frac{\rho_{2}}{\alpha_{2}\left(x^{*}, z^{*}\right)} \geq 0
$$

Then

$$
z^{*}=x^{*}
$$

that is $z^{*}$ is an optimal solution of (WD).

Proof: Suppose that the contradiction is not true, that is $z^{*} \neq x^{*}$. Similar to the proof of Theorem 3.1 we 
obtain

$$
\begin{aligned}
& F\left(x^{*}, z^{*} ; \alpha_{1}\left(x^{*}, z^{*}\right)\left(\sum_{i=1}^{s^{*}} t_{i}^{*} \nabla_{p} w\left(z^{*}, \bar{y}_{i}^{*}, p^{*}\right)+B u^{*}+\lambda^{*} C v^{*}\right)\right) \\
& \geq-\rho_{1} d^{2}\left(x^{*}, z^{*}\right),
\end{aligned}
$$

which implies that

$$
\begin{aligned}
b\left(x^{*}, z^{*}\right) \phi( & \left(\sum_{i=1}^{s^{*}} t_{i}^{*}\left\{f\left(x^{*}, \bar{y}_{i}^{*}\right)-\lambda^{*} h\left(x^{*}, \bar{y}_{i}^{*}\right)\right\}\right. \\
& \left.+x^{* T} B u^{*}+\lambda^{*} x^{* T} C v^{*}\right) \\
& -\left(\sum_{i=1}^{s^{*}} t_{i}^{*}\left\{f\left(z^{*}, \bar{y}_{i}^{*}\right)-\lambda^{*} h\left(z^{*}, \bar{y}_{i}^{*}\right)\right\}\right. \\
& \left.+z^{* T} B u^{*}+\lambda^{*}\left(z^{* T} C v^{*}\right)\right) \\
& \left.-\sum_{i=1}^{s^{*}} t_{i}^{*}\left[w\left(z^{*}, \bar{y}_{i}^{*}, p^{*}\right)-p^{* T} \nabla_{p} w\left(z^{*}, \bar{y}_{i}^{*}, p^{*}\right)\right]\right)
\end{aligned}
$$

$>0$.

From 3)' and (3.13), we can get

$$
\begin{aligned}
& \sum_{i=1}^{s^{*}} t_{i}^{*}\left\{f\left(x^{*}, \bar{y}_{i}^{*}\right)-\lambda^{*} h\left(x^{*}, \bar{y}_{i}^{*}\right)\right\}+x^{* T} B u^{*}+\lambda^{*} x^{* T} C v^{*} \\
& >\sum_{i=1}^{s^{*}} t_{i}^{*}\left\{f\left(z^{*}, \bar{y}_{i}^{*}\right)-\lambda^{*} h\left(z^{*}, \bar{y}_{i}^{*}\right)\right\}+z^{* T} B u^{*} \\
& +\lambda^{*}\left(z^{* T} C v^{*}\right)+\sum_{i=1}^{s^{*}} t_{i}^{*}\left[w\left(z^{*}, \bar{y}_{i}^{*}, p^{*}\right)-p^{* T} \nabla_{p} w\left(z^{*}, \bar{y}_{i}^{*}, p^{*}\right)\right]
\end{aligned}
$$$$
\geq 0 \text {. }
$$

Therefore, following from (3.12) and Lemma 2,

$$
\begin{aligned}
& \sum_{i=1}^{s^{*}} t_{i}^{*}\left[f\left(x^{*}, \bar{y}_{i}^{*}\right)+\left(x^{* T} B x^{*}\right)^{\frac{1}{2}}-\lambda^{*}\left(h\left(x^{*}, \bar{y}_{i}^{*}\right)-\left(x^{* T} C x^{*}\right)^{\frac{1}{2}}\right)\right] \\
& \geq \sum_{i=1}^{s^{*}} t_{i}^{*}\left\{f\left(x^{*}, \bar{y}_{i}^{*}\right)-\lambda^{*} h\left(x^{*}, \bar{y}_{i}^{*}\right)\right\}+x^{* T} B u^{*}+\lambda^{*} x^{* T} C v^{*}
\end{aligned}
$$$$
>0 \text {. }
$$

Since $t^{*}=\left(t_{1}^{*}, t_{2}^{*}, \cdots, t_{s}^{*}\right) \neq 0, t_{i}^{*} \geq 0, i=1,2, \cdots, s^{*}$, $\bar{y}_{i}^{*} \in Y$ and

$h\left(x^{*}, y^{*}\right)-\left(x^{* T} C x^{*}\right)^{\frac{1}{2}}>0, \forall\left(x^{*}, y^{*}\right) \in R^{n} \times R^{l}$, at least exists one $q^{*} \in\{1,2, \cdots, s\}$, such that

$$
\frac{f\left(x^{*}, \bar{y}_{q^{*}}^{*}\right)+\left(x^{* T} B x^{*}\right)^{\frac{1}{2}}}{h\left(x^{*}, \bar{y}_{q^{*}}^{*}\right)-\left(x^{* T} C x^{*}\right)^{\frac{1}{2}}}>\lambda^{*},
$$

which implies that

$$
\sup _{y^{*} \in Y} \frac{f\left(x^{*}, y\right)+\left(x^{* T} B x^{*}\right)^{\frac{1}{2}}}{h\left(x^{*}, y\right)-\left(x^{* T} C x^{*}\right)^{\frac{1}{2}}}>\lambda^{*}
$$

which contradicts with 1$)$ '.

Remark: If we take place condition 2)' of this theorem by

$$
\text { 2)" }\left[\sum_{i=1}^{s^{*}} t_{i}^{*}\left(f\left(\cdot, \bar{y}_{i}^{*}\right)-\lambda^{*} h\left(\cdot, \bar{y}_{i}^{*}\right)\right), \sum_{j=1}^{m} \mu_{j}^{*} g_{j}(\cdot)\right] \text { is higher- }
$$

order $(F, \alpha, \rho, d, b, \phi)_{\mathrm{Bu}^{*}+\lambda^{*} \mathrm{Cv}^{*}}$ vector-pseudoquasi-Type

I at $z^{*}$, and take place condition 3$)^{\prime}$ by

3)" $\phi(a) \geq 0 \Rightarrow a>0, \quad b\left(x^{*}, z^{*}\right)>0$,

$\frac{\rho_{1}}{\alpha_{1}\left(x^{*}, z^{*}\right)}+\frac{\rho_{2}}{\alpha_{2}\left(x^{*}, z^{*}\right)} \geq 0$, the strict converse duality holds too.

\section{Acknowledgements}

This work is supported by Youth Foundation of Beijing University of Technology (X1006011201002).

\section{References}

[1] W. E. Schmitendorf, "Necessary Conditions and Sufficient Conditions for Static Minimax Problems,” Journal of Mathematical Analysis and Applications, Vol. 57, No. 3-4, 1977, pp. 683-693. doi:10.1016/0022-247X(77)90255-4

[2] S. Tanimoto, "Duality for a Class of Nondifferentiable Ma thematical Programming Problems," Journal of Mathematical Analysis and Applications, Vol. 79, No. 2, 1981, pp. 286-294. doi:10.1016/0022-247X(81)90025-1

[3] T. Weir, "Pseudoconvex Minimax Programming," Utilitas Mathematica, Vol. 42, 1992, pp. 234-240.

[4] S. K. Mishra and N. G. Rueda, "Second-Order Duality for Nondifferentiable Minimax Programming Involving Generalized Type I Functions,” Journal of Optimization Theory and Applications, Vol. 130, No. 3, 2006, pp. 477-486. doi:10.1007/s10957-006-9113-9

[5] Z. Husain, A. Jayswal and I. Ahmad, "Second-Order Duality for Nondifferentiable Minimax Programming Problems with Generalized Convexity,” Journal of Glo- bal Optimization, Vol. 44, No. 4, 2009, pp. 593-608. doi:10.1007/s10898-008-9360-4

[6] O. L. Mangasarian, "Second- and Higher-Order Duality in Nonlinear Programming,” Journal of Mathematical Analysis and Applications, Vol. 51, No. 3, 1975, pp. 607-620. doi:10.1016/0022-247X(75)90111-0

[7] J. Zhang, "Generalized Convexity and Higher Order Duality for Mathematical Programming Problem,” Ph.D. Dissertation, La Trobe University, Melbourne, 1998.

[8] S. K. Mishra and N. G. Rueda, "Higher Order Generalized Invexity and Duality in Nondifferentiable Mathe- 
matical Programming,” Journal of Mathematical Analysis and Applications, Vol. 272, No. 2, 2002, pp. 496-506. doi:10.1016/S0022-247X(02)00170-1

[9] I. Ahmad, Z. Huasin and S. Sharma, "Higher-Order Duality in Nondifferentiable Minimax Programming with Generalized Type I Functions,” Journal of Optimization Theory and Applications, Vol. 141, No. 1, 2009, pp. 1-12. doi:10.1007/s10957-008-9474-3

[10] A. Jayswal and I. Stancu-Minasian, "Higher-Order Dual- ity for Nondifferentiable Minimax Programming Problem with Generalized Convexity," Nonlinear Analysis: Theory, Methods \& Applications, Vol. 74, No. 2, 2011, pp. 616-625. doi:10.1016/j.na.2010.09.016

[11] H. C. Lai and J. C. Liu, "Necessary and Sufficient Conditions for Minimax Fractional Programming," Journal of Mathematical Analysis and Applications, Vol. 230, No. 2, 1999, pp. 311-328. doi:10.1006/jmaa.1998.6204 